\title{
Site characterisation at Mount Stromlo: results with a single-detector stereo-SCIDAR
}

\section{Visa Korkiakoski, Doris Grosse, Elliott Thorn, Michael Copeland, Francis Bennet, et al.}

Visa Korkiakoski, Doris Grosse, Elliott Thorn, Michael Copeland, Francis Bennet, James Osborn, Francois Rigaut, Celine d'Orgeville, Marcus Lingham, lan Price, "Site characterisation at Mount Stromlo: results with a single-detector stereo-SCIDAR," Proc. SPIE 10700, Ground-based and Airborne Telescopes VII, 107005B (6 July 2018); doi: 10.1117/12.2313062

Event: SPIE Astronomical Telescopes + Instrumentation, 2018, Austin, Texas, United States 


\title{
Site characterisation at Mount Stromlo: results with a single-detector stereo-SCIDAR
}

\author{
Visa Korkiakoski ${ }^{\mathrm{a}, \mathrm{b}}$, Michael Copeland ${ }^{\mathrm{a}, \mathrm{b}}$, Marcus Lingham ${ }^{\mathrm{a}, \mathrm{b}}$, James Osborn ${ }^{\mathrm{c}}$, Doris Grosse , $^{\mathrm{a}, \mathrm{b}}$, \\ Elliott Thorn ${ }^{\mathrm{a}, \mathrm{b}}$, Francis Bennet ${ }^{\mathrm{a}, \mathrm{b}}$, Ian Price ${ }^{\mathrm{a}, \mathrm{b}}$, Francois Rigaut ${ }^{\mathrm{a}, \mathrm{b}}$, and Celine d'Orgeville ${ }^{\mathrm{a}, \mathrm{b}}$ \\ ${ }^{a}$ Research School of Astronomy and Astrophysics, Australian National University, Mount \\ Stromlo Observatory, Weston Creek, Australia \\ ${ }^{\mathrm{b}}$ SERC Limited, Mount Stromlo, Weston Creek, Australia \\ ${ }^{\mathrm{c}}$ Department of Physics, Centre for Advanced Instrumentation, University of Durham, South \\ Road, Durham DH1 3LE, UK
}

\begin{abstract}
We present the status of the site-characterisation campaign at Mount Stromlo Observatory. The main goal of the project is to aid the development and operation of new adaptive optics (AO) systems for space debris tracking and pushing as well as satellite imaging. The main method we use for the characterisation is based on the SCIntillation Detection And Ranging (SCIDAR) technique. We have designed a unique version of the SCIDAR instrument: a stereo-SCIDAR system that uses a roof prism to separate beams from a double-star system to obtain two isolated pupil images on a single detector. The instrument is installed on the $1.8 \mathrm{~m}$ telescope of Electro-Optic Systems (EOS), sharing facilities with the adaptive optics systems we are currently building. The SCIDAR instrument will be operated intermittently, weather and availability permitting, until sufficient amount of data has been collected to characterise the site. This paper reports the current status of the project: we have recently started the commissioning phase and obtained first measurements with the instrument.
\end{abstract}

Keywords: generalised stereo-SCIDAR, atmospheric turbulence profile measurement, site characterisation, data post-processing

\section{INTRODUCTION}

Adaptive Optics (AO) is a widely used technique to improve the performance of optical instruments suffering the effects of the turbulent atmosphere.

In several applications, it is crucial to know the atmospheric profile, i.e. how the turbulence is distributed as a function of altitude, and how fast it evolves as a function of time. Such applications include, for instance, high-resolution wide-field imaging of celestial objects, ${ }^{1-4}$ ground-to-space optical communication ${ }^{5}$ and proposed orbit perturbation of space debris with a high-power laser. ${ }^{6,7}$ The knowledge of the turbulence profile makes it possible to design optimal AO systems, predict their average long-term performance, and potentially, if the profiling is done in real time, improve their operational performance.

As a partner university of the Space Environment Management Cooperative Research Centre (SERC), the Research School of Astronomy and Astrophysics (RSAA) at the Australian National University (ANU) at Mount Stromlo Observatory is developing AO systems for space situational awareness, specifically space debris tracking and space debris orbit perturbation. ${ }^{6-8}$

As a part of the research efforts, we are implementing a campaign to measure typical profiles of turbulence weight $\left(C_{n}^{2}\right)$ and the winds of atmospheric layers at the Mount Stromlo site. The profiles will be measured by a specifically designed stereo-SCIDAR instrument, ${ }^{8-10}$ a development similar to a previously implemented stereoSCIDAR. ${ }^{11}$ Our design, however, uses alternative relay optics, which makes it possible to build the instrument with a single camera, thus reducing costs and complexity of the project.

Further author information: (Send correspondence to Visa Korkiakoski)

Visa Korkiakoski: E-mail: visa.korkiakoski@anu.edu.au, Telephone: + 61261256084

Ground-based and Airborne Telescopes VII, edited by Heather K. Marshall,

Jason Spyromilio, Proc. of SPIE Vol. 10700, 107005B · C 2018 SPIE

CCC code: $0277-786 \mathrm{X} / 18 / \$ 18 \cdot$ doi: $10.1117 / 12.2313062$

Proc. of SPIE Vol. 10700 107005B-1 
This paper gives an update of our site monitoring project. Section 2 summarises the specifications and design of our stereo-SCIDAR instrument. Section 3 shows the results of our most resent experiments. Finally, conclusions are shown in Section 4.

\section{SPECIFICATIONS AND DESIGN OF MOUNT STROMLO STEREO-SCIDAR}

This section summarises the design of our stereo-SCIDAR instrument. The content is presented here for completeness; our design has been previously reported in our earlier publications..$^{8-10}$

The stereo-SCIDAR was introduced by. ${ }^{11}$ Its theory and principles are discussed in more detailed in numerous earlier publications.

In short, the general SCIDAR images beams (pupil images at a conjugate altitude) from two closely separated stars, as illustrated left in Figure 1. In a stereo-SCIDAR, however, the beams are separated such that the pupil images do not overlap. This can be achieved by two separate detectors (as $\mathrm{in}^{11}$ ) or by inserting a roof prism into the optical path (as in our design ${ }^{8,10}$ ), illustrated right in Figure 1 - we use a double roof prism designed to minimise chromatic aberrations. The imaged beams can then be cross-correlated and processed to obtain vertical turbulence profiles; spatio-temporal cross-correlations will yield the wind profiles as well.

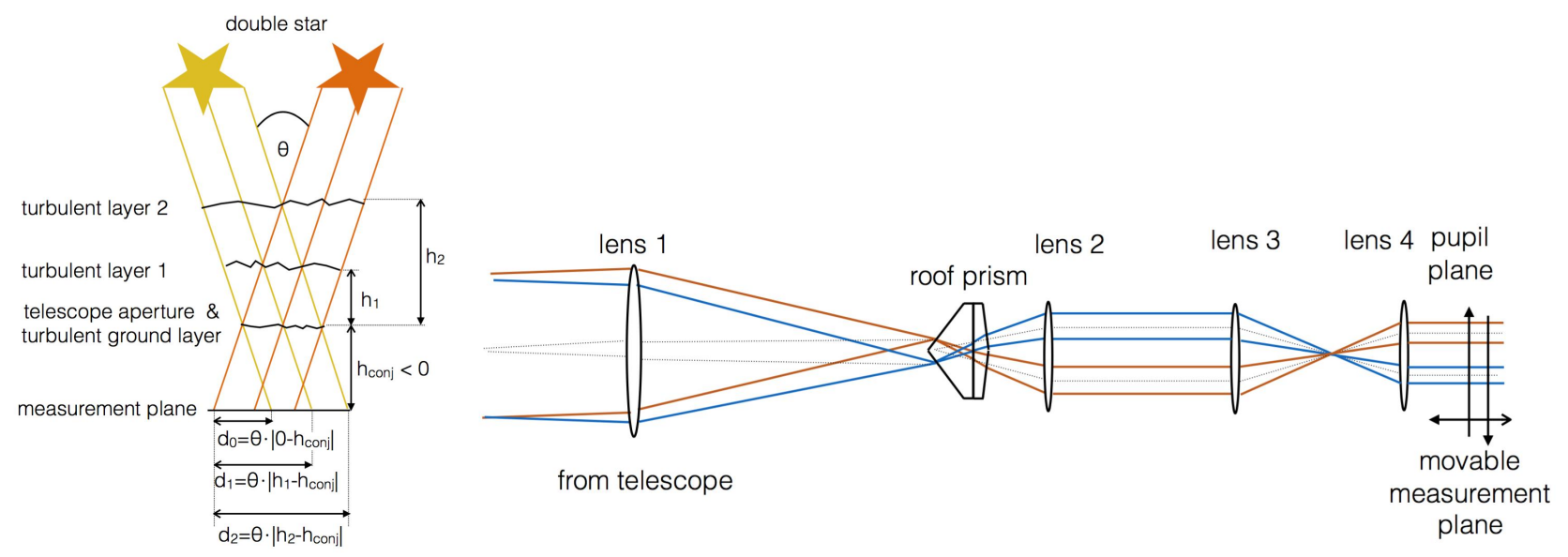

Figure 1: Left: principle of generalised SCIDAR. Phase aberrations from a distinct turbulent layer 1 at height $h_{1}$ through which light shines from a double star with angular separation $\theta$ will be separated by distance $\theta\left|h_{1}-h_{\text {conj }}\right|$. Aberrations from layer 2 will be separated by $\theta\left|h_{2}-h_{\text {conj }}\right|$ and from the ground layer by distance $\theta\left|0-h_{\text {conj }}\right|$. Hence a turbulent layer at height $\mathrm{h}$ will be separated by $\theta\left|h-h_{\text {conj }}\right|$. Right: schematic of optical design of generalised stereo-SCIDAR. The additional roof prism refracts the beams, letting them diverge with the appropriate angle so that the following lens system re-images the scintillation patterns collimated and fully separated. ${ }^{8}$

The design of our stereo-SCIDAR is driven by a need of a good altitude resolution at lower atmosphere while being able to sense the layers up to around $15 \mathrm{~km}$. This is a tradeoff characterised by the equations

$$
h_{\max }=\frac{D-\sqrt{\lambda h_{\text {conj }}}}{\theta},
$$

and

$$
d h=0.78 \frac{\sqrt{\lambda\left|h-h_{\text {conj }}\right|}}{\theta},
$$

where $h_{\max }$ is the maximum altitude where a SCIDAR can characterise a turbulent layer, $d h$ is the altitude resolution, $D$ is the telescope diameter, $h_{\text {conj }}$ is the (negative) conjugation altitude of the detector, $\theta$ is the separation of the used double star, $\lambda$ is the used wavelength, and $h$ is the altitude of a turbulent layer. ${ }^{8,12,13}$ 


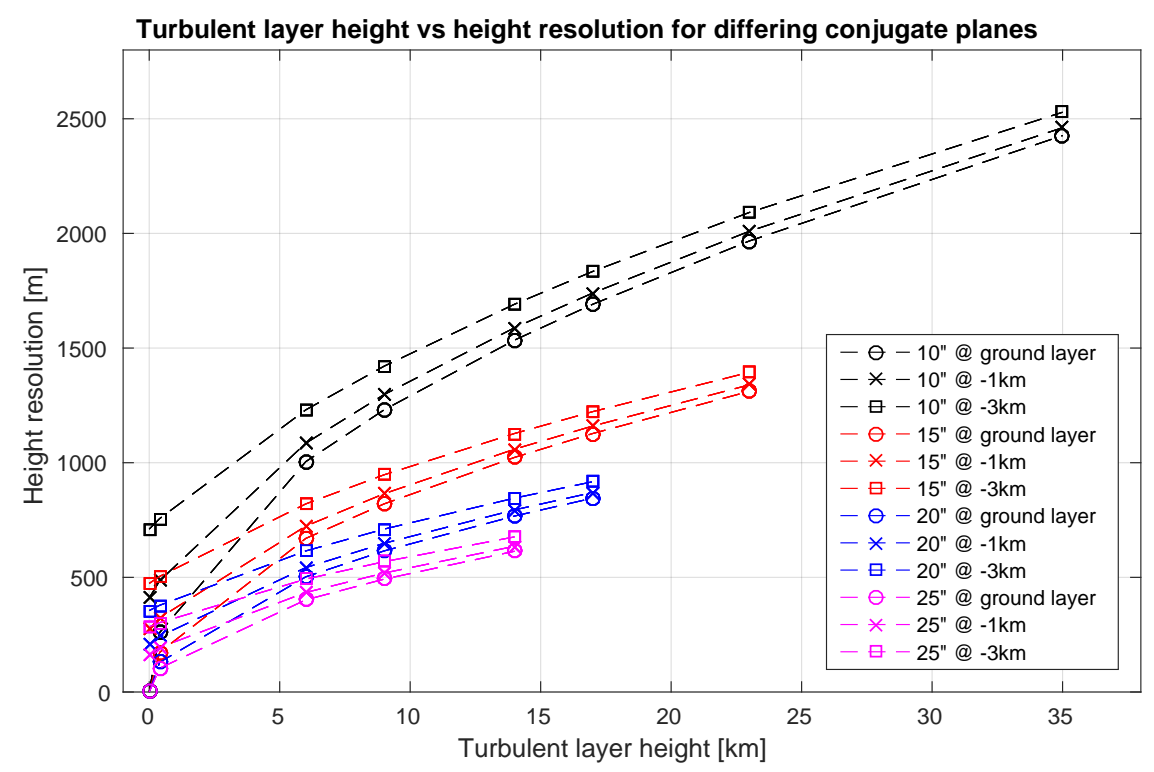

Figure 2: Stereo-SCIDAR resolution as a function of the turbulent layer altitude. Values are for the EOS 1.8 meter telescope at Mount Stromlo site. Four different separations (marked by different colours) and three different conjugate altitudes are shown. At higher altitudes (and lower altitudes when detector at ground layer) the plot is theoretical and in reality the signal-to-noise ratio would be too bad to see peaks in cross-correlations.

The tradeoff is further visualised in Figure 2, which shows the altitude resolution as a function of turbulent layer altitude for separations of $10 "-20$ " and detector altitudes of $0--3 \mathrm{~km}$.

We conclude that to achieve an altitude range of about $15 \mathrm{~km}$, the angular separation between the two stars needs to be 10-20". Larger separations would be preferable to gain more vertical resolution, but a good resolution of 400 to $1000 \mathrm{~m}$ is nonetheless possible.

As a detector, we will use an sCMOS pco.edge camera; the operating wavelength will be 500-700 nm with a variable bandwidth from around 50 to $200 \mathrm{~nm}$. Both beams will be imaged with a resolution of $80 \times 80$ pixels. The camera enables a fast read-out with a noise of about 1 electron. Thus, we will operate the system with a frame-rate of 100-300 $\mathrm{Hz}$ (exposure time set correspondingly to minimise lost photons) to achieve a good temporal resolution and to get images not blurred by the turbulent atmosphere. Ideally, we would prefer readouts closer to $300 \mathrm{~Hz}$ to maximise the temporal resolution. However, when observing dimmer targets, we expect to slow down the rate closer to $100 \mathrm{~Hz}$ to achieve sufficient signal-to-noise ratio.

Figures 3 and 4 show the optical design of the instrument. The collimated beam arrives from EOS $1.8 \mathrm{~m}$ telescope through a $18 \mathrm{~m}$ long coude path, and is downsized by a parabolic beam expander. The beam is then folded, directed through relay lenses and focused on top a roof prism (location shown as a vertical line in the figures). After the prism, the pupil is re-imaged on the detector.

Figure 5 shows a three dimensional model of the SCIDAR instrument. We also illustrate how it is integrated in the coude laboratory on an optical bench shared together with our adaptive optics imager. 


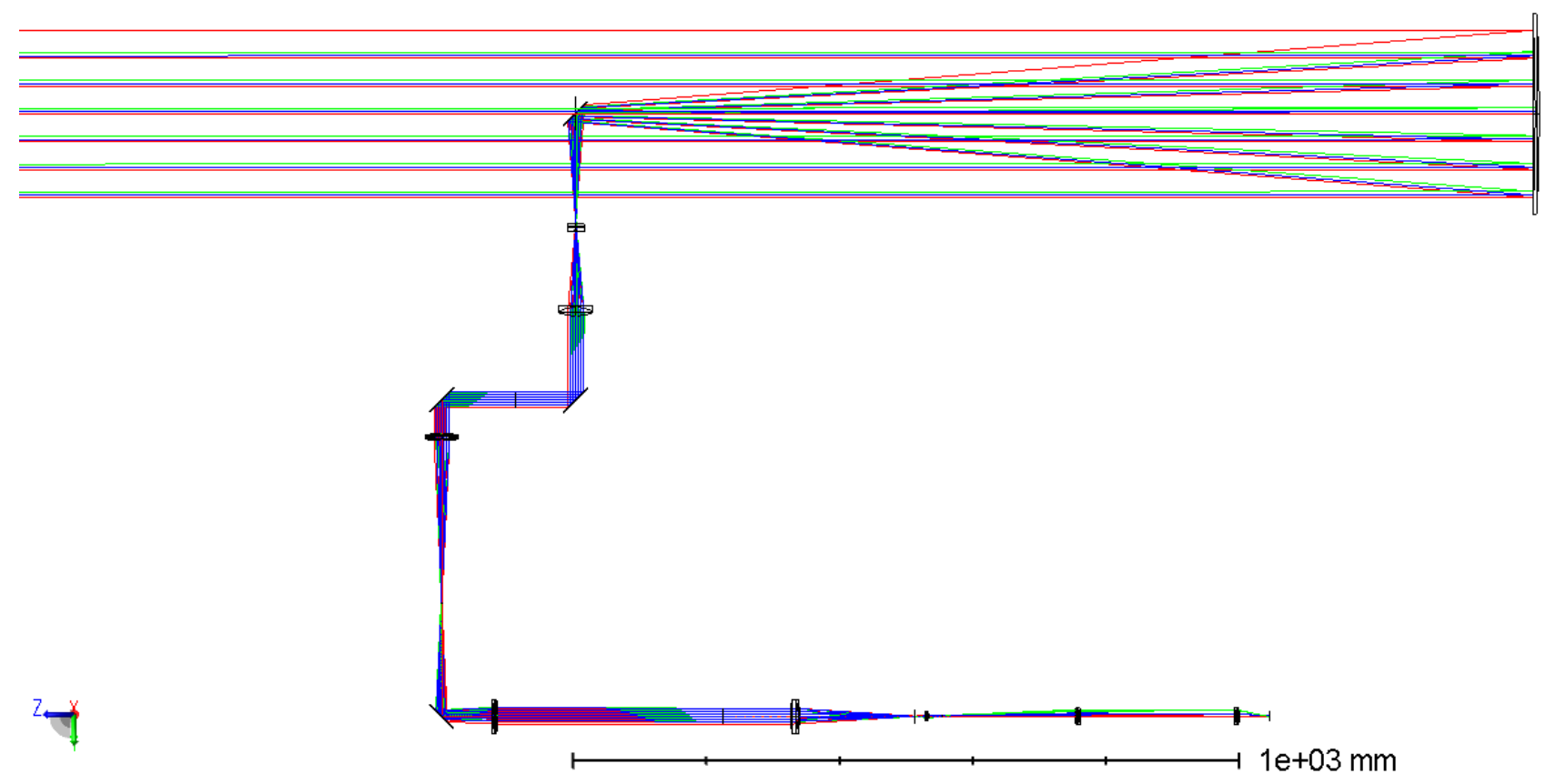

Figure 3: Optical design of our stereo-SCIDAR instrument; an overview. The vertical line in the intermediate focus (before the imaging camera) shows the location where the roof prism is installed.
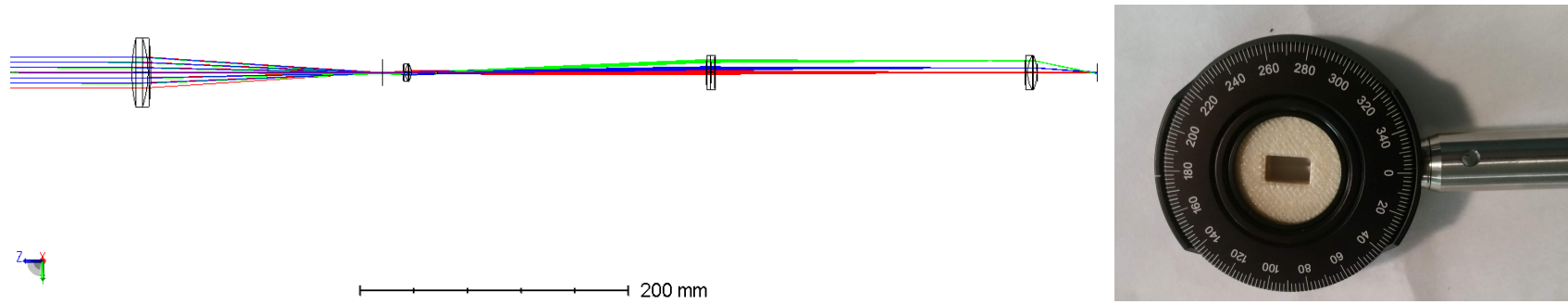

Figure 4: Optical design of our stereo-SCIDAR instrument. Left: a zoom on the imaging arm of the setup. The vertical line in the intermediate focus (before the imaging camera) shows the location where the roof prism is installed. Right: an image of the roof prism, installed on a rotation stage. 

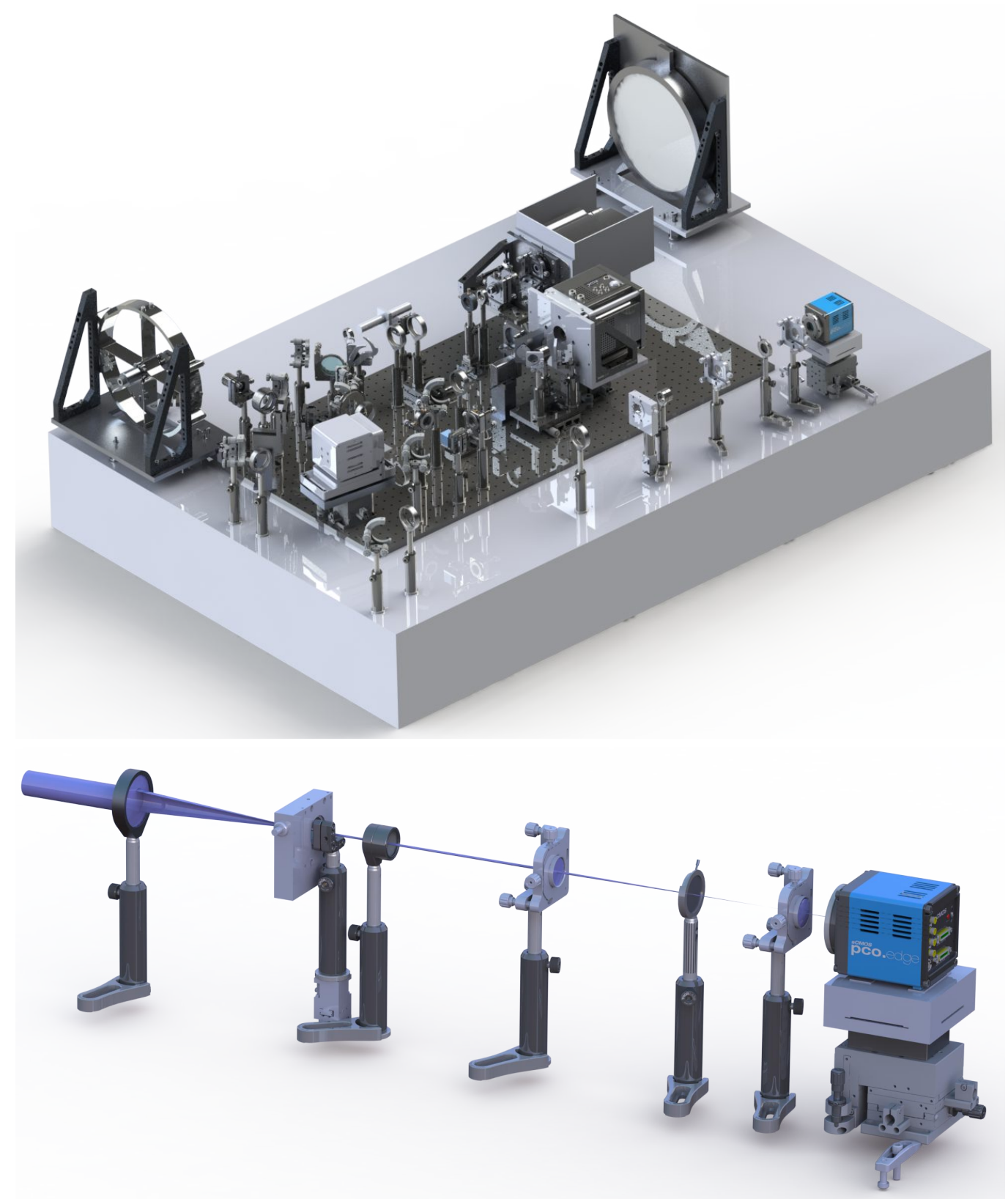

Figure 5: A three dimensional model of the instrument. Top: illustration how the SCIDAR is integrated on the bench hosting our adaptive optics imager. Bottom: a zoom on the stereo-SCIDAR arm of the setup. 


\section{RESULTS}

This section shows the most recent results we have obtained with the stereo-SCIDAR.

We finished the integration of the setup on June 2018. Given non-optimal weather conditions, we are currently able to report successful experiments only for two nights of experiments. These will be referred as trials 1 and 2 in the following.

Results of trial 1 are shown in Figures 6 and 7.
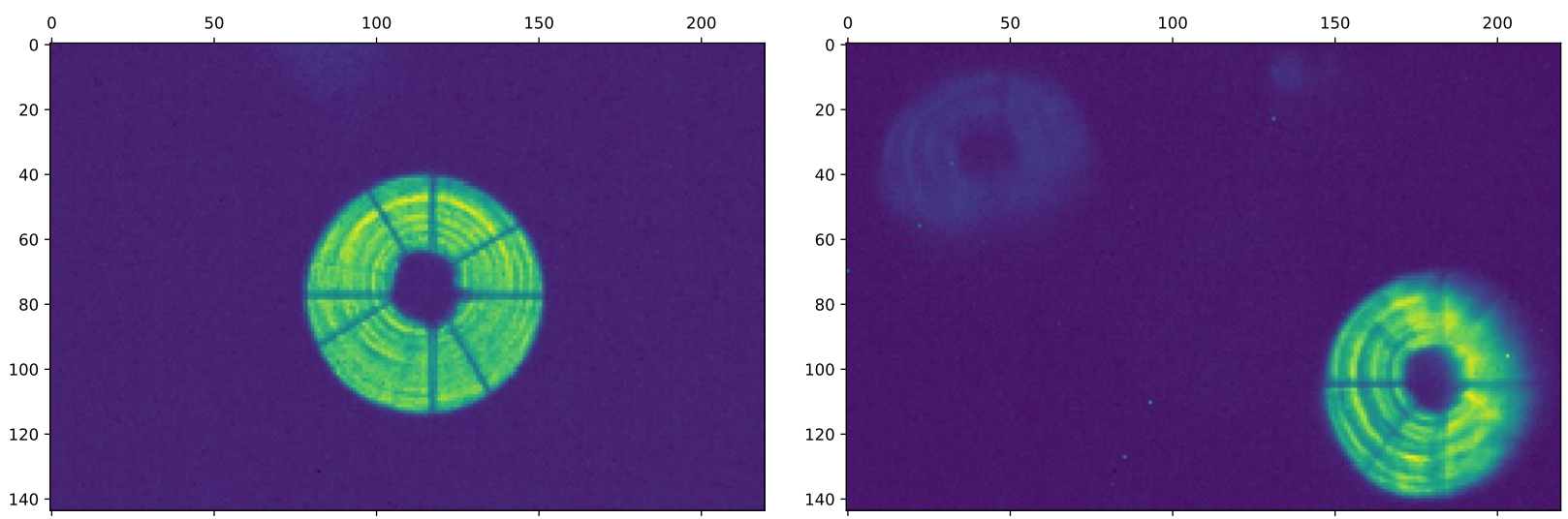

Figure 6: Results of 1st night of experiments. Left: re-imaged pupil of a bright star (no roof prism). Right: re-imaged pupils of a double star (separation 8.6"; magnitudes $3.2,4.1$ ).

At first, we re-imaged the pupil using a single bright star, without the roof prism in the setup. Only the central part of the pupil was imaged; the rest was clipped by an iris at our setup (we would expect to see components shadowing the primary mirror at the pupil border). We also saw several diffraction rings, whose origin is currently being investigated. Possible reasons include errors in optical alignment, aberrations outside the pupil and primary mirror polishing errors.

After pointing the telescope towards a double star (separation 8.6"; magnitudes 3.2, 4.1) and inserting the roof prism, we managed to separate the beams. However, significant parts of the pupils were clipped somewhere along the optical path. We think the most likely source of this clipping is somewhere in the coude path of the telescope.

The first trial was recorded by running the camera at $10 \mathrm{~Hz}$. Post-processing the data using standard approaches and calculating spatio-temporal cross-correlations reveals a trace of signal, as shown in Figure 7. However, bad weather did not permit further experiments to refine the results.

The results from trial 2 are shown in Figures 8 and 9.

We observed a double star with a separation of 7.9 and magnitudes of 4.5 and 6.0. Again, we first looked at the pupil image without the roof prism. Similarly to trial 1, we saw strong diffraction rings and signs that the whole pupil might not be visible.

After inserting the prism, we were able to separate the beams only partially. Based on visual inspection of the acquisition camera, we would have expected the double star to be sufficiently well resolved to be fully separated by the roof prism. However, it turned out to be difficult to align the prism properly to achieve such separation.

In addition, we noticed that significant clipping was still present at the setup, in particular when trying to have both of the pupils re-imaged simultaneously. An attempt was done to manually adjust the telescope pointing, but this provided no significant help.

Nevertheless, we proceeded to record data and post-process it using standard methods. ${ }^{9}$ Figure 9 shows the results. 


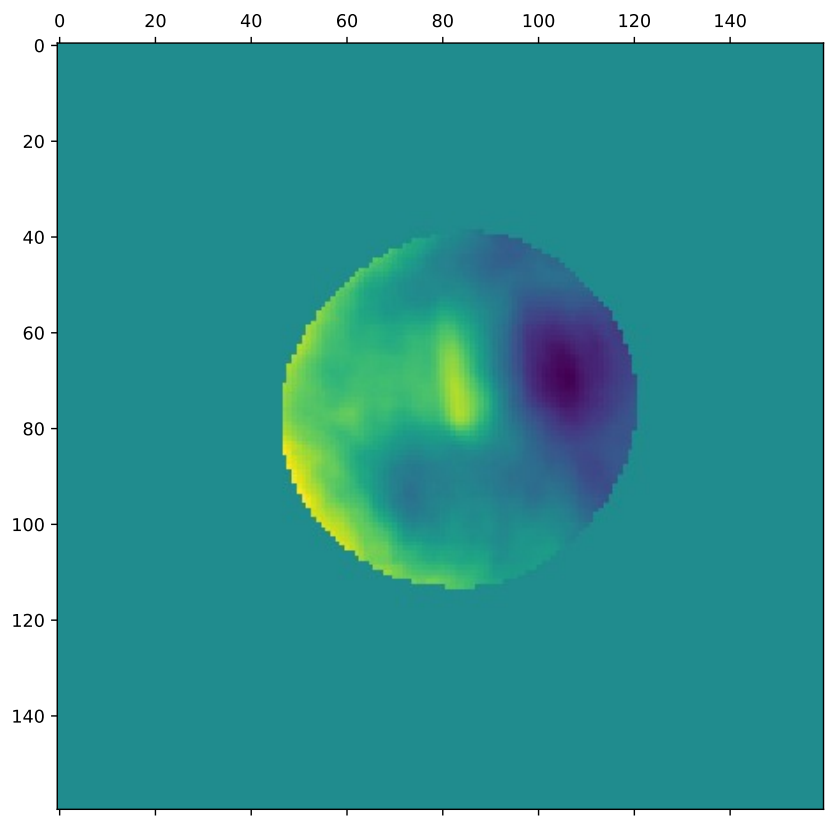

Figure 7: .Trial 1 post-processed: cross-correlation of the pupil images.
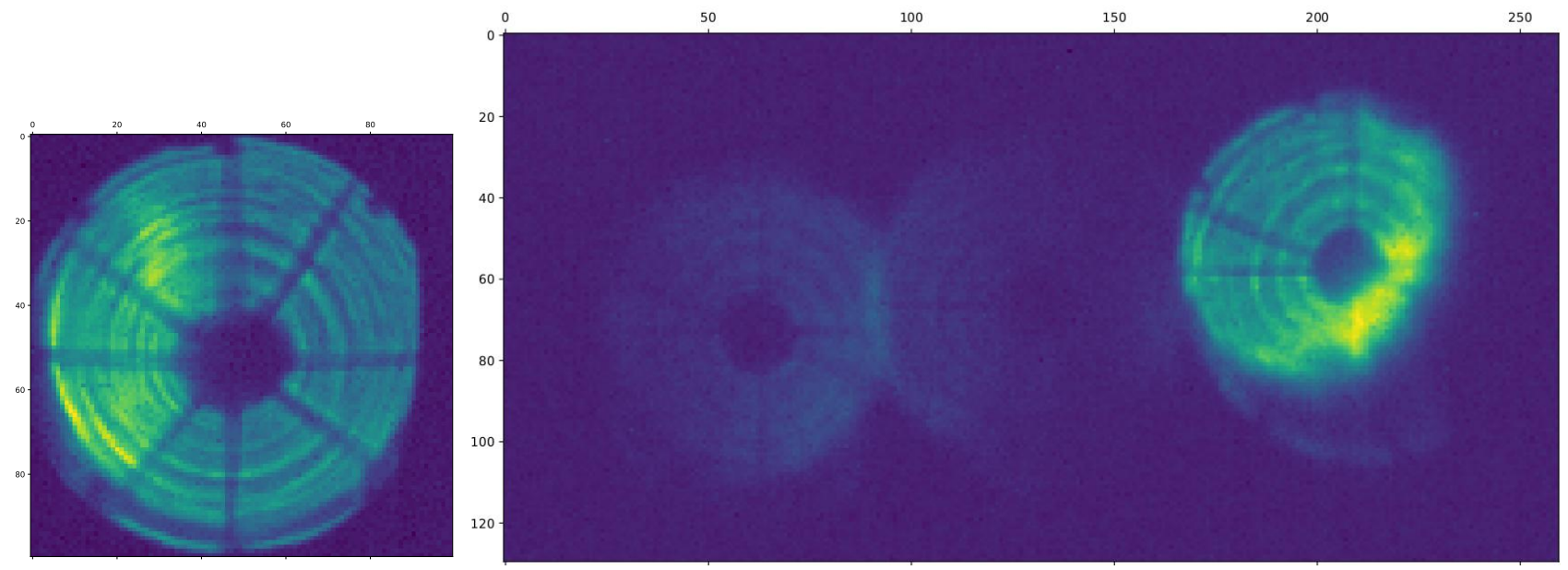

Figure 8: Left: re-imaged pupil of the double star without the roof prism (generalized SCIDAR). Right: partly seprated beam using the roof prism..

We are able to identify at least one strong cross-correlation peak moving in a way that matches the expected signal from an atmospheric layer. The cross-correlation without time difference also shows signs of an expected signal: a strong correlation peak (corresponding to on-ground turbulence) and weaker correlation along the double star position angle (corresponding to turbulence at higher layers).

However, unfortunately, due to various issues still present on the system, the signal is too weak to analyse further and is likely to contain artefacts making more detailed interpretation difficult. 

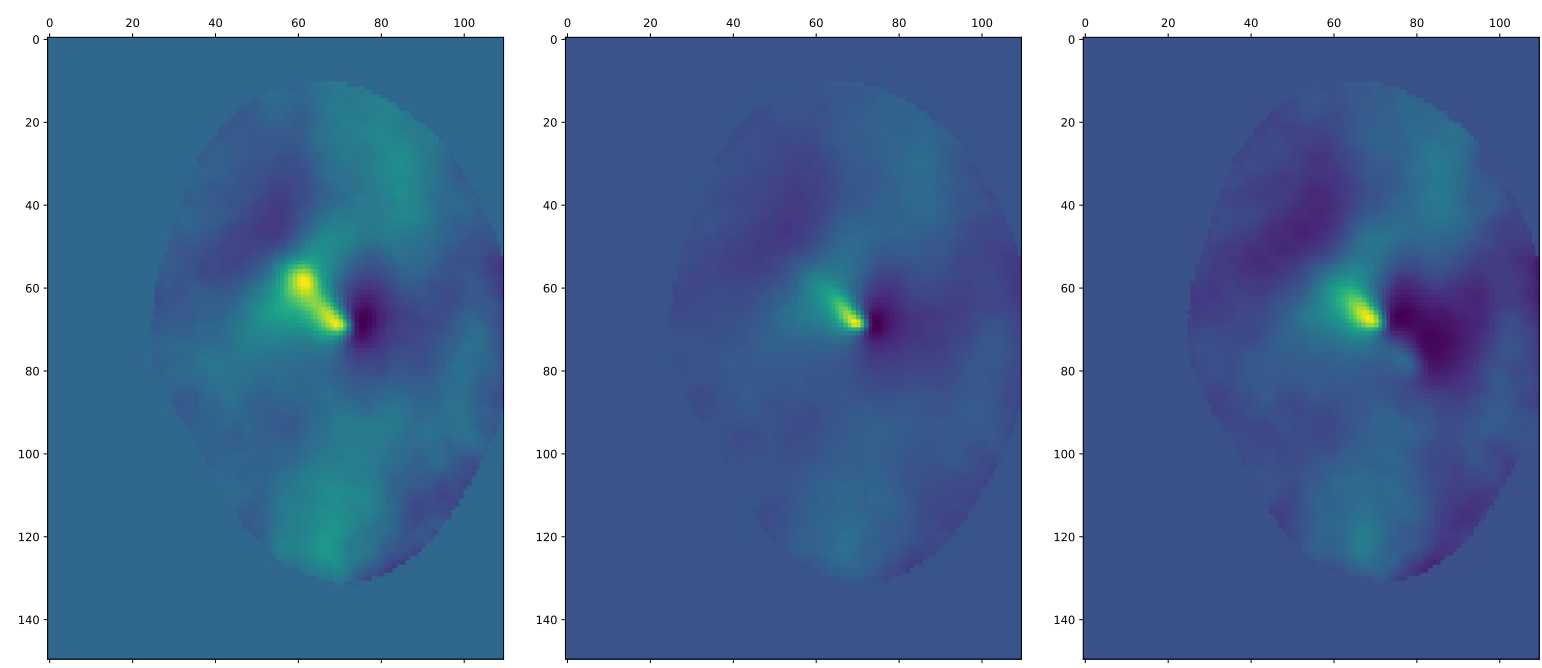

Figure 9: . Trial 2 post-processed: spatio-temporal cross-correlations of the pupil images. Three time differences are shown (camera was running at $100 \mathrm{~Hz}$ ). Left: -5 steps, middle: no time difference, right: 5 steps.

\section{CONCLUSIONS}

We are now in the commissioning phase of a new type of stereo-SCIDAR, designed to operate with the EOS 1.8 $\mathrm{m}$ telescope to characterise the turbulence on Mount Stromlo.

This paper gives the current update of the project.

At the moment, have have been able to run experiments at the telescope only a limited number of nights, mainly due to weather constraints. The experiments indicate that the instrument is likely to perform as planned, but various issues still need to be solved before final conclusions can be made.

The obvious challenges we are currently confronting include:

- imperfect alignment. Due to time constraints, the results presented in this paper were obtained with misaligned optics. Once improved, we expect better signal-to-noise ratio and less blurring in the postprocessed cross-correlation images.

- prism alignment. We are still working on finding a proper solution to adjust the position of the roof prism.

- clipping of the pupils. The clipping is a major challenge, and likely due to a long coude path that limits usable field of view. While aligning the coude optics is likely to improve the situation, a likely long-term solution for the SCIDAR operations will possibly involve accepting a limited amount of vignetting.

In addition, the next steps will involve completing the automation of the data processing pipeline.

\section{ACKNOWLEDGMENTS}

The authors would like to acknowledge the support of the Cooperative Research Centre for Space Environment Management (SERC Limited) through the Australian Government's Cooperative Research Centre Programme.

James Osborn is grateful to the Science and Technology Facilities Committee (STFC) for financial support (grant reference ST/J001236/1 and the research leading to these results has received funding from the European Community's Seventh Framework Programme (FP7/2013-2016) under grant agreement number 312430 (OPTICON). 


\section{REFERENCES}

[1] Le Louarn, M. and Hubin, N., "Wide-field adaptive optics for deep-field spectroscopy in the visible," Monthly Notices of the Royal Astronomical Society 349(3), 1009-1018 (2004).

[2] Beckers, J., "Increasing the size of the isoplanatic patch with multiconjugate adaptive optics??, proceedings eso 30, conference on very large telescopes and their instrumentation, ulrich, mh, ed, 693-703, 1988," Detailed compensation of atmospheric seeing using multiconjugate adaptive optics?, SPIE 1114, 215-17 (1989).

[3] Hammer, F., Sayède, F., Gendron, E., Fusco, T., Burgarella, D., Cayatte, V., Conan, J., Courbin, F., Flores, H., Guinouard, I., et al., "Scientific drivers for eso future vlt/vlti instrumentation," in [ESO Workshop], 139 (2001).

[4] Assémat, F., Gendron, E., and Hammer, F., "The falcon concept: multi-object adaptive optics and atmospheric tomography for integral field spectroscopy-principles and performance on an 8-m telescope," Monthly Notices of the Royal Astronomical Society 376(1), 287-312 (2007).

[5] Chan, V. W., "Free-space optical communications," Journal of Lightwave Technology 24(12), 4750-4762 (2006).

[6] Bennet, F., Conan, R., D’Orgeville, C., Murray, M., Paulin, N., Price, I., Rigaut, F., Ritchie, I., Smith, C., and Uhlendorf, K., "Adaptive optics for laser space debris removal," in [SPIE Astronomical Telescopes+ Instrumentation], 844744-844744, International Society for Optics and Photonics (2012).

[7] Bennet, F., D'Orgeville, C., Price, I., Rigaut, F., Ritchie, I., and Smith, C., "Adaptive optics for satellite imaging and space debris ranging," in [Proceedings of the Advanced Maui Optical and Space Surveillance Technologies Conference], Ryan, S., ed., 1, 2, The Maui Economic Development Board (2015).

[8] Grosse, D., Bennet, F., Korkiakoski, V., Rigaut, F., and Thorn, E., "Single detector stereo-scidar for mount stromlo," in [Proc. SPIE], 99093D-99093D (2016).

[9] Korkiakoski, V., Osborn, J., Grosse, D., Thorn, E., Piatrou, P., Bennet, F., and Rigaut, F., "Single detector stereo-scidar for mount stromlo: data analysis," in [Proc. SPIE], 99790L-99790L (2016).

[10] Thorn, E., Korkiakoski, V., Grosse, D., Bennet, F., Rigaut, F., and dOrgeville, C., "Stereo-scidar system for improvement of adaptive optics space debris-tracking activities," in [AMOS conference], (2017).

[11] Shepherd, H., Osborn, J., Wilson, R., Butterley, T., Avila, R., Dhillon, V., and Morris, T., "Stereo-scidar: optical turbulence profiling with high sensitivity using a modified scidar instrument," Monthly Notices of the Royal Astronomical Society 437(4), 3568-3577 (2013).

[12] Prieur, J. L., Daigne, G., and Avila, R., "Scidar measurements at pic du midi," Astronomy \& Astrophysics 371(1), 366-377 (2001).

[13] Osborn, J., Wilson, R. W., Dhillon, V., Avila, R., and Love, G., "Conjugate-plane photometry: reducing scintillation in ground-based photometry," Monthly Notices of the Royal Astronomical Society 411(2), 1223$1230(2011)$. 